\title{
Téoros
}

Revue de recherche en tourisme

\section{Fondements pour l'analyse du tourisme en Charlevoix}

\section{Philippe Dubé}

Volume 17, numéro 1, printemps 1998

Le tourisme dans un Pays à part : Charlevoix

URI : https://id.erudit.org/iderudit/1072368ar

DOI : https://doi.org/10.7202/1072368ar

Aller au sommaire du numéro

Éditeur(s)

Université du Québec à Montréal

ISSN

0712-8657 (imprimé)

1923-2705 (numérique)

Découvrir la revue

Citer ce document

Dubé, P. (1998). Fondements pour l'analyse du tourisme en Charlevoix. Téoros,

17(1), 4-4. https://doi.org/10.7202/1072368ar d'utilisation que vous pouvez consulter en ligne.

https://apropos.erudit.org/fr/usagers/politique-dutilisation/ 
pas très attentives aux nouvelles atractions. excepté le Casino et répondent peri aux efforts consentis pour les atriver en toutes saisons.

L'analyse de Jean Stafford et Bruno Sarrasin jette un nouvel eclairage sur les tendances de toffre el de la demande touristiques en Charlevoix depuis $10-12$ ans. On retient surtout la pression de l'ofje, evalaée par le nombre de chambres disponibles, par saison et chaque anné. Les responsables du tourisme ont investi sur thebergement, en priorite; is portent maintenant lew attention sur les atractions dans l'espoir de recevoir el de retenir davantage de touristes. Cest le defi le plus grand, nous semble-t-il.

Le casino de Charlevoix, ouveri depuis quatre ans à peine, constitue ume nouvelle expérience pour le tourisme en région et c'est au pays dAlexis le Trotieur que se fait cet essai! Fantil le voir comme ane attraction de plas, sim- plement comme un elargissement de la gamme des activités qui peuvent satisfaire les tourisles? Claude Gingras er Lise Lapointe ne le concoivent pas ainsi: leur texte est un essai qui rend plausible ane hypothese: les clientèles intéressées par le casino sont spécifiques; elles accordent peud'anention à l'ensemble des attractions qui caracterisent Charlevoix et se mélangent peu aux autres touristes. Cette réflexion est stimulante mais laisse entière la question de l'accroissemen sowhaitable de la demande touristique ${ }^{2}$.

On saluera enfin le texte vibrant de Rejean Tremblay dit Lagadelle: il raconte une histoire de vie, un amour du pays natal et we decouverte dis lourisme comme on en lit rarement. Puisque le développenent towristique ne naît pas spontanement, la culture touristique a besoin de temps et d'ouverture d'esprit powr fleurir.

\section{NOTES}

1 Voir le comple-rendu, par Claude Moulin. du livre de Jean DesGagniers (1995), Charlevoir, wa paysenchome, Suinte-Foy, Presses de l'Université Laval,

2 Ce texte est un cssail, redisons-le. Les auteurs ont tirê l'essentiel de leurs idécs d'observations de longue durée sur le terrain du casino, complétées par quelques entrevues semi-dirigkes ivec des agents touristiques. Faut-il dire qu'une enquête sous forme de sondage serait la bienvenue sur toutes les questions soulevere dans ce texte?

\section{FONDEMENTS POUR L'ANALYSE DU TOURISME EN CHARLEVOIX}

\section{Philippe Dubé, professeur Département d'histoire - Université Laval}

Le regard historien, pour y allerd"entre de jeu avec she evidence, nous ramène constamment a faire l'étude d'un sujet donné par l'examen étiologique de ce qui l'a précédé. En fait. nous tirons de la sorte les enseignements que nous livre le passé dans le bat de mieur comprendre le présent. Et du coup, nous sommes enclin à croire que la conscience historigue est garante de l'avenis; comme si le futur avait, pour ainsi dire, besoin du passe. C'est ce que nous allons tenter de metre en pratique sommairement, fort de l'exencice studieur que nous avons fait ces demieres années de la villegiature dans Charlevoix.

En effet, l'étude de cetre longue tradition vieille de plus de deux cents ans - faut-il te rappeler - nous instrait sur les fondements de la frequentation de plaisance dans cette region a partir d'un constat bien simple: le succes touristique de Charlevoix s'est joué, se joue. et se jouera probablement encone longtemps. sur denx elements essentiels, l'environnemen et l'equipement. Il s'agit donc maintenant de savoir comment s'opere une telle dynamique binaire et d'en faire un bref énoncé en guise d'introduction à ce numéro tout spécial.

D'abord lenvironnement doit être ici compris conme un des ingredients de base à une pareille composition. Quand on dit environnement, on ne fait pas uniquement référence à la geographie belle et immense d'une contrée que l'on a souvent associée à la Suisse du Quebec. Il wa sans dire que la rencontre de la mer naissante et des montagnes laurentiennes offre un coup d'oeil ine galable qui a longtemps fait te bonhear des paysagistes tant liftéraires que visuels, Mais il faut aussi noter que les aspects de la présence humaine ont enrichi et continuent d'enrichir les points de vue recherches par les touristes avec ses villages en enfilade, les prés déchiquetés par les clotares de percher et la volumétrie capricieuse di băti qui ponctue à sa manière les vastes horizons. L'activite humaine est donc ici par. the intergante de loffre qui est faite aux visi. teurs curiax de decouvir, non seulement la démesure d'un paysage surdimensionné mais aussi le coractère spécifique qu' a profilé la culture de ce terroir. En somme, nous devons dès lors parler plutôt de patrimoine régional, à la fois naturel el culurel, venant definir les contenus du produit touristique à développer dans un marche deja fort encombré. C'est d'alleurs ce qui a établi sa spécificité dans l'atribution du statu tant convoité de Réserve mondiale de la biosphère de Charlevoix du seul fait que lactivite humaine a té prise en compte dans la considération globale de cet environnement parfaitement exceptionnel. Voilà qui peut être indicateur d'un certain développement d'avenir.

Puis le deuxieme element dont on ne peut pas faire l'économie si l'on veut révéler un tant soit peu ane dimension touristique qui vaille, c'est bien entendu f'existence d'un équipement adequat à rendre certe acrivite possible. II faus tout de suite signaler que cet aute ingrédient a éte de tout temps remarquable dans Charlevoix sil'on tient compte de ce qui se trouvait en ces mêmes termes dans les autres ré. gions du Quebec. Par exemple, fa nature des equipements de transport a longtemps permis un acces privilégé à une région qui se transforme magiquement, sous l'effet de surprise, en un pays merveilleux. Le réseau routier d'aujourd' hui a certes quelque peu banalise la dramaturgie des décors charlevoisiens d"antan, mais il reste des vues nombreases et inprenables sur ses routes sinueuses que plus d'unte region touristique lat envie torjours. De plus, il existe une infrastructure hoteliere que les transporteurs maritimes ont d'abord largement financée afin de doter la région d" un équipement d'accueil encore difficile à égaler quand on est loin des centres urbains. S'ajoutent à ces outils indispensables pour recevoir des clienteles de plas en plus variées. des installations culturelles de mieur en mieux arriculés qui donnen un sens véritable à la visite de ces lieux empreints d histoire et de proverbiale cretativite. Faut-il ajouter à cette panoplie la bonne able qui doit faire parte des attirails de base d'une région qui se veut pour le moins invitante. En effet, l'émergence des produits régionaux d'appellation contrólée vient confirmer cente orientation sur le long chemin de lexcellence. Et à cesujet, plusieurs restaurateurs, producteurs et eleveurs sont déjà engagés dans cette voie parfois hasardease mais combien nécessaive au success touristique diune région.

Parions donc que les deur éléments qui viennent constituer l'assise du troisième siècle de lactivité touristique dans Charlevoix feront en sorte que cette activité sera constinue du tardem synchronisé d'un equipement a la meswe d'un exvironnenent patrimonial grandiose. 